\title{
Efeitos da luz e da temperatura na germinação de sementes de Eremanthus (Asteraceae), ocorrentes na Serra do Cipó, MG, Brasil ${ }^{1}$
}

\author{
Simone Batalha Velten ${ }^{2}$ e Queila Souza Garcia ${ }^{2,3}$
}

Recebido em 08/06/2004. Aceito em 23/03/2005

\begin{abstract}
RESUMO - (Efeitos da luz e da temperatura na germinação de sementes de Eremanthus (Asteraceae), ocorrentes na Serra do Cipó, MG, Brasil). Estudos de germinação são importantes para a propagação de plantas, regeneração e conservação de comunidades vegetais. $O$ objetivo deste trabalho foi avaliar a viabilidade e o comportamento germinativo de sementes de Eremanthus elaeagnus (Mart. ex DC.) Schultz-Bip, E. glomerulatus Less e E. Incanus (Less.) Less. A viabilidade das sementes foi verificada através do teste de tetrazólio. Os testes de germinação foram realizados sob luz e escuro contínuos em temperaturas constantes de $15,20,25,30,35$ e $40^{\circ} \mathrm{C}$ e em temperaturas alternadas (fotoperíodo de $12 \mathrm{~h}$ ) de 15-25, 15-30, 15-35, 20-30, 20-35 e 25-35 ${ }^{\circ} \mathrm{C}$. A viabilidade das sementes variou significativamente entre populações e diferentes anos de coleta. As percentagens de germinação das sementes foram sempre baixas, devido, principalmente, à ausência de embriões. Sementes de E. elaeagnus germinaram nas temperaturas de 15 a $30^{\circ} \mathrm{C}$, de E. glomerulatus de 20 a $30{ }^{\circ} \mathrm{C}$ e E. incanus de 15 a $35^{\circ} \mathrm{C}$. As três espécies germinaram na luz e no escuro, apresentando diferenças significativas entre estes dois regimes apenas nas temperaturas de 25 e $35^{\circ} \mathrm{C}$ (E. incanus) e $30^{\circ} \mathrm{C}$ (E. glomerulatus). Em comparação com as outras espécies Eremanthus incanus apresentou maiores percentagens de germinação em faixa de temperatura mais ampla e suas sementes são mais rápidas para germinar.
\end{abstract}

Palavras-chave: Asteraceae, Eremanthus, germinação, viabilidade, luz e temperatura

\begin{abstract}
Light and temperature effects in the germination of seeds of Eremanthus (Asteraceae) that occur in Serra do Cipó, Minas Gerais State, Brazil). Studies of germination are important for the propagation of plants, and the regeneration and the preservation of plant communities. The purpose of this study was to evaluate the viability and the germinative behavior of the seeds of Eremanthus elaeagnus (Mart. ex DC.) Schultz-Bip, E. glomerulatus Less. and E. incamus (Less) Less. The viability of seeds was confirmed through the tetrazolium test. Germination tests were conducted under continuous light and darkness at constant temperatures of 15, 20, 25, 30, 35 and $40^{\circ} \mathrm{C}$ and at alternated temperatures (photoperiod of 12 hours) range from 15-25, 15-30, 15-35, 20-30, 20-35 and 25-35 ${ }^{\circ} \mathrm{C}$. Seed viability varied significantly among years and populations. The germination percentage was always low, due mainly to absence of embryos. The seeds of E. elaeagnus germinated at temperatures from 15 to $30^{\circ} \mathrm{C}$; E. glomerulatus at temperatures from 20 to $30^{\circ} \mathrm{C}$; and E. incamus at temperatures from 15 to $35^{\circ} \mathrm{C}$. The three species germinated in light and darkness, presenting significant differences between these treatments only at temperatures of 25 and $35^{\circ} \mathrm{C}($ E. incanus $)$ and $30^{\circ} \mathrm{C}($ E. glomerulatus $)$. Eremanthus incanus presented a higher germination percentage over a wider temperature range, and its seeds germinated faster than E. elaeagnus and E. glomerulatus.
\end{abstract}

Keywords: Asteraceae, Eremanthus, germination, viability, light and temperature

\section{Introdução}

Estudos sobre a biologia das sementes são essenciais para o conhecimento da estrutura e da dinâmica das comunidades vegetais devido à importância das mesmas para a propagação de plantas e regeneração de florestas (Vázquez-Yanes \& OrozcoSegovia 1996). O conhecimento sobre o comportamento germinativo das sementes é fundamental para a utilização de espécies nativas na reabilitação de áreas degradadas e constitui providência essencial para proteger as espécies contra a ameaça de extinção (Labouriau 1983).

Os fatores ambientais mais importantes para a regulação da germinação são temperatura, luz e umidade do solo (Baskin \& Baskin 1988). O efeito da luz e da temperatura nas sementes varia grandemente entre diferentes espécies e populações (Pons 1992; Bewley \& Black 1994). Para cada população de sementes há uma faixa característica de temperatura na qual a germinação ocorre (Bewley \& Black 1994), sendo que algumas sementes ou frutos não germinam

\footnotetext{
1 Parte da dissertação de Mestrado da primeira Autora

2 Laboratório de Fisiologia Vegetal, Departamento de Botânica, Instituto de Ciências Biológicas, Universidade Federal de Minas Gerais, C. Postal 486, CEP 30161-970, Belo Horizonte, MG, Brasil

3 Autores para correspondência: svelten@terra.com.br; queila@netuno.lcc.ufmg.br
} 
em temperaturas constantes e outras germinam melhor em temperaturas alternadas (Thompson 1974).

Na família Asteraceae são numerosos os estudos sobre germinação de espécies invasoras (Corkidi et al. 1991; Ferreira et al. 2001; Figueiredo 1975; Garcia \& Sharif 1995; Gomes \& Fernandes 2002; Ladeira et al. 1987; Schütz \& Milberg 1997; Tamashino \& Leitão Filho 1978), medicinais (Afolayan et al. 1997; Ferreira et al. 2001; Ladeira et al. 1987) e ornamentais (Bunker 1994; Owens \& Call 1985). Entretanto, poucos estudos foram realizados com espécies desta família em risco de extinção (Clampitt 1987; Falkner et al. 1997; Jusaitis et al. 2004; Walker \& Powell 1999) ou com espécies típicas do cerrado (Cesarino \& Zaidan 1998; Felippe \& Silva 1984; Maluf 1993; Ruggiero \& Zaidan 1997).

O gênero Eremanthus, pertencente à tribo Vernonieae, compreende 18 espécies de árvores e arbustos distribuídos nos domínios dos cerrados brasileiros, principalmente em Minas Gerais, Goiás e Bahia (MacLeish 1987). Em Minas Gerais, as espécies de Eremanthus, localmente chamadas de "candeias", estão submetidas a constante pressão exploratória, sendo seus caules freqüentemente utilizados como mourões de cerca (MacLeish 1987). Estas plantas são também utilizadas para a obtenção de óleo essencial, devido à presença de lactonas sesquiterpênicas, geralmente isoladas de suas partes aéreas, que possuem propriedades citotóxica, antitumoral e antimicrobiana (Bohlmann et al. 1980; Picman 1986).

Na Serra do Cipó, Eremanthus elaeagnus, E. glomerulatus e E. incanus são freqüentemente encontradas formando grandes populações que podem dominar ambientes de cerrado e de campo rupestre. Estas espécies encontram-se na Lista das Espécies Presumivelmente Ameaçadas de Extinção da Flora de Minas Gerais, uma vez que não há informações suficientes para uma conclusão segura sobre o seu real status de ameaça, mas há indícios que permitem considerá-las como tal (Mendonça \& Lins 2000).

A finalidade deste estudo foi avaliar a influência da luz e da temperatura na germinação e investigar a viabilidade das sementes de E. elaeagnus, E. glomerulatus e E. incanus, visando obter dados sobre a capacidade germinativa destas espécies e assim subsidiar o planejamento de estratégias para a conservação das mesmas.

\section{Material e métodos}

Foram coletados aquênios de 50 indivíduos de cada espécie, na Serra do Cipó, Minas Gerais, no período de junho/2001 a julho/2003. O clima da Serra do Cipó é tropical de altitude, com temperaturas médias anuais variando entre 17 e $18,5^{\circ} \mathrm{C}$ e a precipitação média anual entre 1.450 e $1.850 \mathrm{~mm}$, sendo que as chuvas ocorrem principalmente entre dezembro e março (Antunes 1986). Os aquênios de Eremanthus elaeagnus (C. Martius ex DC.) Schultz-Bip. foram coletados em duas áreas de campo cerrado

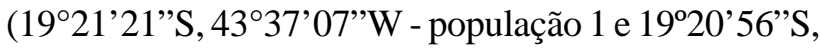
4338'04”'W - população 2), E. glomerulatus Less. de uma população em campo cerrado (19 $17^{\prime} 57^{\prime}$ 'S e 4336'06”'W) e E. incanus (Less.) Less. numa região

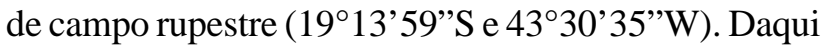
em diante aquênios serão referidos como sinônimos de sementes, uma vez que são funcionalmente análogos a estas (Fenner 1983).

Para cada espécie foram medidas quatro amostras de 25 sementes sem papus (comprimento e largura), com o auxílio de paquímetro digital. A massa de matéria seca $(\mathrm{mg})$ das sementes foi obtida pela pesagem de quatro amostras (25 sementes sem papus), em balança analítica, após secagem em estufa $\left(105^{\circ} \mathrm{C}\right)$ durante $24 \mathrm{~h}$. Nos dois casos, os papus foram retirados para efeito de comparação com a maioria dos trabalhos existentes (Leishman et al. 2001).

Foram feitas contagens de sementes predadas, murchas e aparentemente viáveis em quatro repetições de 25 glomérulos de E. elaeagnus e E. glomerulatus, coletados em agosto e julho/2001, respectivamente. Após as contagens, foram calculadas as médias de cada repetição e os erros padrões. Não foram realizadas contagens para $E$. incanus, uma vez que suas sementes não apresentavam sinais de murcha e predação facilmente visíveis.

Para a realização dos experimentos foram selecionadas sementes que não apresentavam sinais de predação ou murcha. Dentre as sementes aparentemente viáveis, não foi possível diferenciar aquelas que possuíam embriões das que não possuíam. Uma vez que a cavidade seminal de grande parte dos aquênios sem embrião é preenchida por tecido parenquimático e que o pericarpo é esclerificado (S.B. Velten, dados não publicados), não se pode distinguir as sementes com ou sem embrião através de pressão, transparência ou morfologia externa.

Os testes de germinação foram realizados utilizando-se placas de Petri de $9 \mathrm{~cm}$ diâm. contendo folha dupla de papel filtro umedecida com solução de nistatina -100 U.I./L- (Lemos Filho et al. 1997), sendo utilizadas 4 repetições de 25 sementes recém-coletadas por tratamento. As placas foram colocadas sob luz 
branca fluorescente $\left(30 \mu \mathrm{mol} . \mathrm{m}^{-2} \cdot \mathrm{s}^{-1}\right)$ e escuro contínuos, sendo mantidas nas temperaturas de 15, 20, $25,30,35$ e $40{ }^{\circ} \mathrm{C}$. Os testes de alternância de temperatura foram realizados a 15-25, 15-30, 15-35, 20-30, 20-35 e $25-35^{\circ} \mathrm{C}$, fotoperíodo de $12 \mathrm{~h}$, sendo o período de luz coincidente com a temperatura mais alta de cada par. Os tratamentos de escuro foram feitos envolvendo-se as placas de Petri em papel alumínio e mantendo-as em sacos pretos de polietileno. A contagem da germinação foi realizada diariamente, até a estabilização da resposta (30 dias para E. elaeagnus e E. incanus e 40 dias para E. glomerulatus), sendo que as sementes mantidas no escuro foram observadas em ambiente escuro, sob luz verde de segurança. O critério adotado para o início da germinação foi a emergência da radícula.

A viabilidade foi verificada através do teste de tetrazólio (Delouche et al. 1962), utilizando-se sementes recém-coletadas, sem sinais de predação ou murcha. Foram utilizadas quatro repetições de 30 sementes previamente embebidas em água destilada e mantidas em solução de tetrazólio (1\%), durante 24 horas, a $25^{\circ} \mathrm{C}$. Foram consideradas viáveis as sementes que apresentaram embrião corado de vermelho.

A velocidade de germinação foi estimada através do cálculo do tempo médio de germinação (Labouriau 1983). Como os dados obtidos apresentaram desvios padrões com variação errática, não sujeitos a normalização, procedeu-se a análise não paramétrica de Kruskal-Wallis, cujas ordenações médias foram então comparadas com o teste t, ao nível significância de 5\% (Sampaio 2002).

\section{Resultados}

As medidas de comprimento e largura e os valores de massa das sementes estão apresentados na Tabela 1. Sementes de E. glomerulatus apresentaram as maiores medidas de comprimento, largura e massa seca, seguidas por E. elaeagnus e E. incanus. Eremanthus elaeagnus apresentou menores taxas de predação e de sementes murchas, resultando em uma maior percentagem de sementes aparentemente viáveis, em relação a E. glomerulatus (Tab. 1).

As sementes de E. elaeagnus germinaram nas temperaturas constantes entre 15 e $30^{\circ} \mathrm{C}$, não havendo diferenças significativas entre as percentagens de germinação obtidas na luz e no escuro, dentro da mesma temperatura (Tab. 2). Não ocorreu germinação em 35 e $40{ }^{\circ} \mathrm{C}$. A germinação na luz se iniciou a partir do décimo primeiro dia $\left(20^{\circ} \mathrm{C}\right)$ e no escuro a partir do nono dia $\left(30^{\circ} \mathrm{C}\right)$. As velocidades de germinação foram sempre baixas, sem diferenças significativas entre os tratamentos (Tab. 2).

Em comparação com as temperaturas constantes, os tratamentos com temperaturas alternadas não promoveram a germinação de E. elaeagnus (Tab. 2). A maior percentagem de germinação (10\%) foi obtida na alternância de $15-30{ }^{\circ} \mathrm{C}$ e a menor (1\%) na de 25-35 ${ }^{\circ} \mathrm{C}$, sendo significativa a diferença entre estes dois pares de temperatura. Não ocorreu germinação em $15-35^{\circ} \mathrm{C}$. Na alternância de $20-30^{\circ} \mathrm{C}$ a velocidade média de germinação foi significativamente maior que em $15-25$ e $25-35^{\circ} \mathrm{C}$ (Tab. 2).

As sementes de E. glomerulatus germinaram na luz, nas temperaturas constantes de 20 a $30{ }^{\circ} \mathrm{C}$, sem diferenças significativas (Tab. 3). No escuro ocorreu germinação entre 15 e $30{ }^{\circ} \mathrm{C}$, sendo que a $15^{\circ} \mathrm{C}$ a percentagem de germinação obtida (1\%) foi significativamente inferior que nas demais temperaturas ( 7 a 12\%). Em todos os tratamentos as percentagens de germinação foram sempre muito baixas, não ultrapassando $12 \%$. Na temperatura de $30{ }^{\circ} \mathrm{C}$ a germinação foi significativamente maior no escuro. $\mathrm{O}$ início da germinação ocorreu a partir do $19^{\circ}$ dia e a velocidade média de germinação foi baixa em todos os tratamentos, sem diferenças significativas entre luz e escuro (Tab. 3).

Os tratamentos com temperaturas alternadas não promoveram a germinação de E. glomerulatus, em comparação com as temperaturas constantes (Tab. 3). Somente $2 \%$ das sementes germinaram na alternância de $15-30{ }^{\circ} \mathrm{C}$ e $4 \%$ na de $20-30{ }^{\circ} \mathrm{C}$. Nas demais alternâncias não ocorreu germinação.

Tabela 1. Comprimento (C), largura (L), massa de matéria seca (MS), percentagem de sementes predadas (SP), murchas (SM) e aparentemente viáveis (SAV) de Eremanthus spp. coletadas na Serra do Cipó, Minas Gerais, Brasil. Média \pm erro-padrão. $(\mathrm{N}=4 \times 25)$

\begin{tabular}{|c|c|c|c|c|c|c|}
\hline Espécies & $\mathrm{C}(\mathrm{mm})$ & $\mathrm{L}(\mathrm{mm})$ & MS (mg) & $\mathrm{SP}(\%)$ & $\mathrm{SM}(\%)$ & SAV (\%) \\
\hline E. elaeagnus & $2,96 \pm 0,11$ & $1,06 \pm 0,02$ & $0,71 \pm 0,06$ & $3,50 \pm 2,53$ & $26,82 \pm 5,40$ & $69,68 \pm 6,12$ \\
\hline E. glomerulatus & $3,14 \pm 0,04$ & $1,38 \pm 0,03$ & $1,65 \pm 0,11$ & $10,63 \pm 0,03$ & $33,47 \pm 0,06$ & $55,98 \pm 0,09$ \\
\hline E. incanus & $2,40 \pm 0,06$ & $0,68 \pm 0,04$ & $0,23 \pm 0,02$ & - & - & - \\
\hline
\end{tabular}


Tabela 2. Características germinativas de sementes de Eremanthus elaeagnus (Mart. ex. DC.) Schultz-Bip (população 2), sob temperaturas constantes e alternadas (fotoperíodo de 12 h). G - Percentagem de germinação; VM - Velocidade média de germinação e IG - Início da germinação. Letras minúsculas comparam colunas e letras maiúsculas linhas. Valores seguidos por letras iguais não diferem entre si. $(\mathrm{N}=4 \times 25$; duração do experimento $=30$ dias. $)$

\begin{tabular}{|c|c|c|c|c|c|c|c|}
\hline \multirow{2}{*}{$\begin{array}{c}\text { Temperatura } \\
\left({ }^{\circ} \mathrm{C}\right)\end{array}$} & \multicolumn{2}{|c|}{$\mathrm{G}(\%)$} & \multicolumn{3}{|c|}{ VM (dias) } & \multicolumn{2}{|c|}{ IG (dias) } \\
\hline & Luz & Escuro & Luz & & Escuro & Luz & Escuro \\
\hline 15 & $14 \mathrm{aA}$ & $22 \mathrm{aA}$ & 0,035 & $\mathrm{aA}$ & 0,031 aA & 13 & 16 \\
\hline 20 & $12 \mathrm{aA}$ & $11 \mathrm{abA}$ & 0,043 & $\mathrm{aA}$ & 0,027 aA & 11 & 15 \\
\hline 25 & $12 \mathrm{aA}$ & $5 \mathrm{bcA}$ & 0,037 & $\mathrm{aA}$ & 0,031 aA & 17 & 22 \\
\hline 30 & $9 \mathrm{aA}$ & $11 \mathrm{abA}$ & 0,042 & $\mathrm{aA}$ & 0,052 aA & 13 & 9 \\
\hline 35 & $0 \mathrm{bA}$ & $0 \mathrm{cA}$ & & - & - & - & - \\
\hline 40 & $0 \mathrm{bA}$ & $0 \mathrm{cA}$ & & - & - & - & - \\
\hline $15-25$ & \multicolumn{2}{|c|}{$5 \mathrm{abc}$} & \multicolumn{3}{|c|}{$0,023 \mathrm{~b}$} & \multicolumn{2}{|c|}{13} \\
\hline $15-30$ & \multicolumn{2}{|c|}{$10 \mathrm{a}$} & \multicolumn{3}{|c|}{$0,043 \mathrm{ab}$} & \multicolumn{2}{|c|}{13} \\
\hline $15-35$ & \multicolumn{2}{|c|}{$0 \mathrm{c}$} & \multicolumn{3}{|c|}{-} & \multicolumn{2}{|c|}{-} \\
\hline $20-30$ & \multicolumn{2}{|c|}{$8 \mathrm{a}$} & \multicolumn{3}{|c|}{$0,056 \mathrm{a}$} & \multicolumn{2}{|c|}{8} \\
\hline $20-35$ & \multicolumn{2}{|c|}{$7 \mathrm{ab}$} & \multicolumn{3}{|c|}{$0,042 \mathrm{ab}$} & \multicolumn{2}{|c|}{13} \\
\hline $25-35$ & \multicolumn{2}{|c|}{$1 \mathrm{bc}$} & \multicolumn{3}{|c|}{$0,011 \mathrm{~b}$} & \multicolumn{2}{|c|}{23} \\
\hline
\end{tabular}

Tabela 3. Características germinativas de sementes de Eremanthus glomerulatus Less., sob temperaturas constantes e alternadas (fotoperíodo de 12 h). G - Percentagem de germinação; VM - Velocidade média de germinação e IG - Início da germinação. Letras minúsculas comparam colunas e letras maiúsculas linhas. Valores seguidos por letras iguais não diferem entre si. $(\mathrm{N}=4 \times 25$; duração do experimento $=40$ dias $)$.

\begin{tabular}{|c|c|c|c|c|c|c|c|c|}
\hline \multirow{2}{*}{$\begin{array}{c}\text { Temperatura } \\
\left({ }^{\circ} \mathrm{C}\right)\end{array}$} & \multicolumn{3}{|c|}{$\mathrm{G}(\%)$} & \multicolumn{3}{|c|}{ VM (dias) } & \multicolumn{2}{|c|}{ IG (dias) } \\
\hline & Luz & & Escuro & Luz & Escuro & & Luz & Escuro \\
\hline 15 & $0 \mathrm{bA}$ & & $1 \mathrm{bA}$ & - & 0,007 & $\mathrm{~b}$ & - & 37 \\
\hline 20 & $6 \mathrm{aA}$ & & $12 \mathrm{aA}$ & $0,020 \mathrm{aA}$ & 0,029 & $a b A$ & 25 & 30 \\
\hline 25 & $7 \mathrm{aA}$ & & 7 aA & $0,016 \mathrm{aA}$ & 0,030 & $\mathrm{aA}$ & 29 & 23 \\
\hline 30 & $5 \mathrm{aB}$ & & $10 \mathrm{aA}$ & $0,018 \mathrm{aA}$ & 0,034 & $\mathrm{aA}$ & 19 & 21 \\
\hline 35 & $0 \mathrm{bA}$ & & $0 \mathrm{bA}$ & - & - & & - & - \\
\hline 40 & $0 \mathrm{bA}$ & & $0 \mathrm{bA}$ & - & - & & - & - \\
\hline $15-25$ & \multicolumn{3}{|c|}{$0 \mathrm{a}$} & \multicolumn{3}{|c|}{ - } & \multicolumn{2}{|c|}{ - } \\
\hline $15-30$ & \multicolumn{3}{|c|}{$2 \mathrm{a}$} & \multicolumn{3}{|c|}{$0,035 \mathrm{a}$} & \multicolumn{2}{|c|}{26} \\
\hline $15-35$ & \multicolumn{3}{|c|}{$0 \mathrm{a}$} & \multicolumn{3}{|c|}{-} & \multicolumn{2}{|c|}{-} \\
\hline $20-30$ & \multicolumn{3}{|c|}{$4 \mathrm{a}$} & \multicolumn{3}{|c|}{$0,038 \mathrm{a}$} & \multicolumn{2}{|c|}{21} \\
\hline $20-35$ & \multicolumn{3}{|c|}{$0 \mathrm{a}$} & \multicolumn{3}{|c|}{-} & \multicolumn{2}{|c|}{-} \\
\hline $25-35$ & \multicolumn{3}{|c|}{$0 \mathrm{a}$} & \multicolumn{3}{|c|}{-} & \multicolumn{2}{|c|}{ - } \\
\hline
\end{tabular}

As sementes de E. incanus germinaram na luz e no escuro, nas temperaturas entre 15 e $35^{\circ} \mathrm{C}$ (Tab. 4). Na luz o percentual de germinação a $35^{\circ} \mathrm{C}(26 \%)$ foi significativamente inferior ao obtido nas demais temperaturas (40 a $51 \%)$, exceto a $20^{\circ} \mathrm{C}(37 \%)$. No escuro o percentual de germinação a $35^{\circ} \mathrm{C}(1 \%)$ também foi significativamente inferior ao obtido nas demais temperaturas (25 a $40 \%$ ). Não ocorreu germinação a $40{ }^{\circ} \mathrm{C}$. Em 25 e $35^{\circ} \mathrm{C}$ as percentagens de germinação foram significativamente maiores na luz do que no escuro. Na luz, as velocidades médias de germinação obtidas a 20,25 e $30^{\circ} \mathrm{C}$ foram significativamente maiores que nas demais temperaturas (Tab. 4). No escuro, as maiores velocidades médias foram obtidas a 20 e $25{ }^{\circ} \mathrm{C}$ (Tab. 4). A $15{ }^{\circ} \mathrm{C}$ a velocidade média de germinação foi significativamente maior no escuro, enquanto a $35^{\circ} \mathrm{C}$ foi maior na luz. A germinação se iniciou a partir do segundo dia na luz e no escuro, em temperaturas constantes (Tab. 4).

Sob temperaturas alternadas as percentagens de germinação variaram de 5 a $17 \%$, sendo que na alternância de $15-35{ }^{\circ} \mathrm{C}$ a germinação foi significati- 
Tabela 4. Características germinativas de sementes de Eremanthus incanus (Less.) Less, sob temperaturas constantes e alternadas (fotoperíodo de 12 h). G - Percentagem de germinação; VM - Velocidade média de germinação e IG - Início da germinação. Letras minúsculas comparam colunas e letras maiúsculas linhas. Valores seguidos por letras iguais não diferem entre si. ( $\mathrm{N}=4 \times 25$; duração do experimento $=30$ dias $)$.

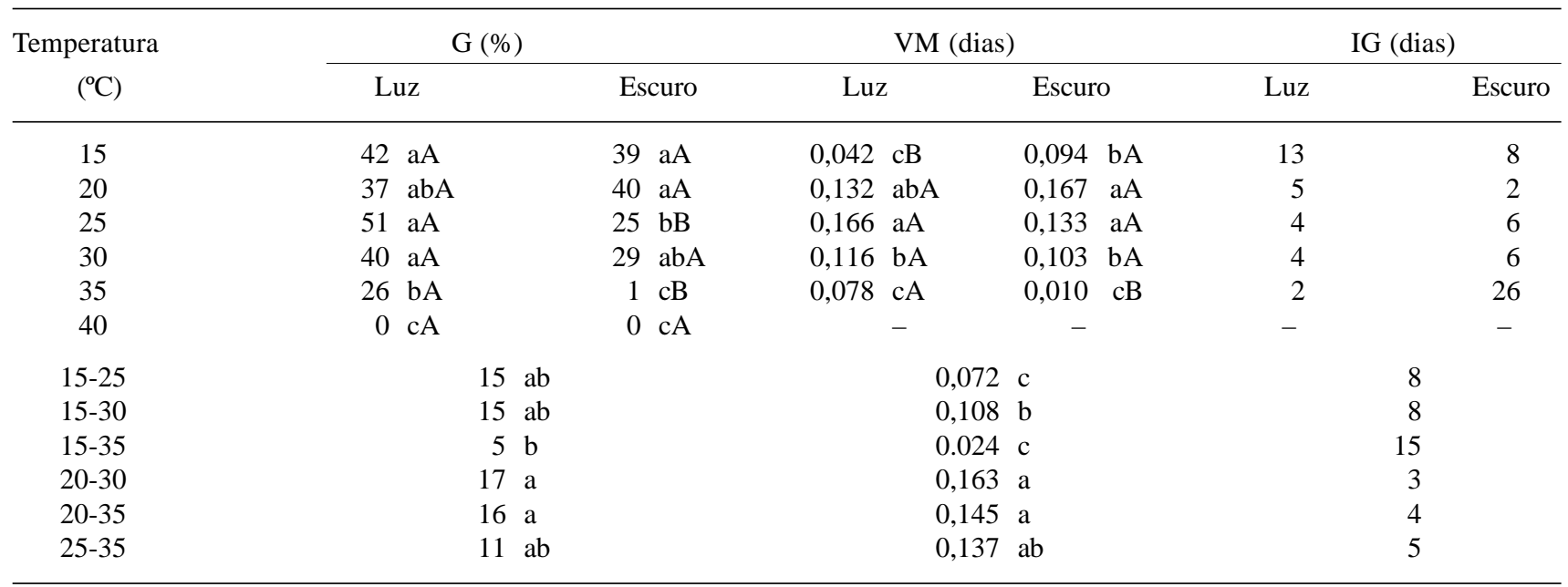

vamente inferior a $20-30$ e $20-35^{\circ} \mathrm{C}$ (Tab. 4). As maiores velocidades de germinação foram obtidas nas temperaturas de $20-30,20-35$ e $25-35^{\circ} \mathrm{C}$, sem diferenças significativas entre estas alternâncias. A germinação de sementes de $E$. incanus se iniciou a partir do terceiro dia em temperaturas alternadas (Tab. 4).

Os experimentos com sementes de E. incanus sob temperaturas alternadas foram realizados em 2002 e as sementes produzidas neste ano apresentaram viabilidade inferior àquelas coletadas em 2001 (ver
Tab. 5), com as quais foram realizados os experimentos com temperaturas constantes. Para efeito de comparação, foi estimada a proporção de sementes germinadas em relação à viabilidade obtida através do teste com tetrazólio e verificou-se que aproximadamente $100 \%$ das sementes viáveis de E. incanus germinaram na temperatura constante de $25^{\circ} \mathrm{C}$ e nas alternâncias de 15-25, 15-30, 20-30 e 20-35 ${ }^{\circ} \mathrm{C}$.

Eremanthus incanus apresentou maiores percentagens de germinação e também maiores

Tabela 5. Sementes viáveis de Eremanthus spp., com base no teste de tetrazólio, coletadas na Serra do Cipó, Minas Gerais, Brasil. Os testes estatísticos comparam colunas e diferentes amostras de uma mesma espécie. Valores seguidos por letras iguais não diferem entre si. ( $\mathrm{N}=4 \times 30)$.

\begin{tabular}{|c|c|c|c|}
\hline Espécie/população & Data da coleta & Sementes viáveis (\%) & Sementes sem embrião (\%) \\
\hline $\begin{array}{l}\text { E. elaeagnus } \\
\text { (população 1) }\end{array}$ & $\begin{array}{l}12 / 08 / 2001 \\
15 / 09 / 2001 \\
04 / 07 / 2002 \\
06 / 08 / 2002 \\
28 / 07 / 2003\end{array}$ & $\begin{array}{ll}5,83 & \mathrm{~b} \\
3,33 & \mathrm{~b} \\
5,83 & \mathrm{~b} \\
5,83 & \mathrm{~b} \\
8,33 & \mathrm{~b}\end{array}$ & $\begin{aligned} 91,67 & \text { a } \\
90,00 & \text { a } \\
90,93 & \text { a } \\
89,17 & \text { a } \\
83,33 & \text { ab }\end{aligned}$ \\
\hline $\begin{array}{l}\text { E. elaeagnus } \\
\quad \text { (população 2) }\end{array}$ & $\begin{array}{l}06 / 08 / 2002 \\
28 / 07 / 2003\end{array}$ & $\begin{array}{rl}25,83 & \mathrm{a} \\
3,33 & \mathrm{~b}\end{array}$ & $\begin{array}{l}67,50 \text { b } \\
84,17 \text { ab }\end{array}$ \\
\hline E. glomerulatus & $\begin{array}{l}16 / 06 / 2001 \\
04 / 07 / 2001 \\
12 / 08 / 2001 \\
15 / 09 / 2001 \\
04 / 07 / 2002 \\
06 / 08 / 2002 \\
28 / 07 / 2003\end{array}$ & $\begin{array}{l}21,66 \mathrm{ab} \\
16,66 \mathrm{ab} \\
16,66 \mathrm{ab} \\
13,33 \mathrm{bc} \\
18,33 \mathrm{ab} \\
22,50 \mathrm{ab} \\
24,99 \mathrm{a}\end{array}$ & $\begin{array}{l}72,50 \mathrm{abc} \\
73,33 \mathrm{ab} \\
79,17 \mathrm{a} \\
80,00 \mathrm{a} \\
79,17 \mathrm{a} \\
52,50 \mathrm{c} \\
60,83 \mathrm{bc}\end{array}$ \\
\hline E. incanus & $\begin{array}{l}28 / 10 / 2001 \\
04 / 11 / 2002\end{array}$ & $\begin{array}{l}50,83 \text { a } \\
14,17 \text { b }\end{array}$ & $\begin{array}{l}49,17 \text { b } \\
83,33 \text { a }\end{array}$ \\
\hline
\end{tabular}


velocidades médias de germinação, quando comparado com E. elaeagnus e E. glomerulatus (Tab. 2, 3 e 4). A $40{ }^{\circ} \mathrm{C}$ não ocorreu germinação para nenhuma das três espécies. A germinação de E. glomerulatus ocorreu numa faixa de temperatura mais estreita, enquanto as sementes de E. incanus germinaram em ampla faixa de temperatura (Tab. 2, 3 e 4).

Os testes mostraram que 3 a $8 \%$ das sementes de E. elaeagnus da população 1 eram viáveis, sem diferenças significativas entre os períodos amostrados (Tab. 5). As sementes da população 2, coletadas em 2002, apresentaram viabilidade significativamente superior $(25,83 \%)$, sendo os testes de germinação realizados com as sementes deste lote.

A viabilidade das sementes de E. glomerulatus variou de 13 a $25 \%$ (Tab. 5). Sementes de E. incanus apresentaram viabilidade de 50 e $14 \%$ em 2001 e 2002, respectivamente (Tab. 5). Foi observado que cerca de 50 a $90 \%$ das sementes inviáveis das três espécies não possuíam embriões (Tab. 5).

\section{Discussão}

O tamanho da semente é característica que ocupa posição central na ecologia das espécies (Leishman et al. 2001). Em geral, sementes com massa menor que 0,1 $\mathrm{mg}$ apresentam necessidade de luz e temperaturas alternadas para a germinação, enquanto sementes com massa igual ou maior que $1,0 \mathrm{mg}$ não apresentam estes requerimentos (Grime et al. 1981; Thompson \& Grime 1983). O significado ecológico da relação entre o tamanho da semente e o requerimento de luz e flutuação de temperaturas parece estar ligado à necessidade de evitar a germinação em locais muito profundos no solo, onde há dificuldade para as sementes pequenas emergirem (Pons 1992; Thompson \& Grime 1983). As três espécies de Eremanthus apresentam sementes relativamente pequenas, entretanto, com massa seca maior que $0,1 \mathrm{mg}$ (Tab. 1 ). As temperaturas alternadas não promoveram a germinação das espécies de Eremanthus estudadas, assim como observado para outras espécies de Asteraceae (Corkidi et al. 1991; Randi \& Felippe 1981; Schütz \& Milberg 1997). As percentagens de germinação obtidas neste estudo foram semelhantes na luz e no escuro. Eremanthus incanus, espécie com sementes menores $(0,23 \mathrm{mg})$, foi a única que apresentou valores de germinação significativamente maiores na luz, nas temperaturas de 25 e $35^{\circ} \mathrm{C}$ (Tab. 4).

O fotoblastismo positivo foi observado em diversas espécies de Asteraceae ocorrentes em regiões tropicais, sendo a maioria herbáceas e invasoras (Afolayan et al.
1997; Corkidi et al. 1991; Ferreira et al. 2001; Figueiredo 1975; Garcia \& Sharif 1995; Ladeira et al. 1987; Maluf \& Wizentier 1998; Radford \& Cousens 2000; Ruggiero \& Zaidan 1997; Sassaki et al. 1999). Entretanto, poucos estudos com Asteraceae mostram comportamento germinativo em relação à luz semelhante ao observado para as espécies de Eremanthus estudadas. Baccharis dracunculifolia, Asteraceae arbustiva que tem ocorrência no cerrado, apresentou percentagens de germinação a $15^{\circ} \mathrm{C}$ sem diferenças significativas entre luz e escuro, enquanto nas demais temperaturas a percentagem de germinação obtida na luz foi significativamente maior que no escuro (Gomes \& Fernandes 2002), semelhantemente a E. incanus (Tab. 4). Launaea arborescens, um arbusto de região semi-desértica, apresentou comportamento afotoblástico (Schütz \& Milberg 1997), similar ao observado para $E$. eleagnus e E. glomerulatus (Tab. 2 e 3).

A percentagem de germinação tende a ser maior em temperaturas correspondentes ao período do ano cujas condições ambientais são favoráveis à emergência e ao estabelecimento da plântula (Baskin \& Baskin 1988; Bell et al. 1993). Para as espécies de Erementhus estudadas não foi possível estabelecer faixa de temperatura ótima para a germinação, uma vez que os percentuais de germinação foram, em geral, muito baixos. Entretanto, foi possível observar que as sementes de E. glomerulatus (Tab. 3) possuem exigências mais específicas, germinando em faixa de temperatura mais estreita $\left(20\right.$ a $\left.30{ }^{\circ} \mathrm{C}\right)$, as sementes de E. elaeagnus (Tab. 2) germinaram em faixa de temperatura intermediária $\left(15\right.$ a $\left.30{ }^{\circ} \mathrm{C}\right)$, enquanto as sementes de $E$. incanus (Tab. 4) germinaram em faixa de temperatura mais ampla $\left(15\right.$ a $\left.35^{\circ} \mathrm{C}\right)$.

O tempo médio (ou velocidade) de germinação é bom índice para avaliar a rapidez de ocupação de uma espécie em um determinado ambiente (Ferreira et al. 2001). A germinação rápida é característica de espécies cuja estratégia é se estabelecer no ambiente o mais rápido possível ou quando oportuno, aproveitando condições ambientais favoráveis, como, por exemplo, a formação de clareiras ou ocorrência de chuvas. Em contrapartida, a germinação rápida pode ser imprópria ao estabelecimento de uma espécie quando a germinação ocorrer, por exemplo, em resposta a uma chuva errática e isolada na estação seca (Borghetti \& Ferreira 2004). Segundo Ferreira et al. 2001, quanto ao tempo médio de germinação as sementes podem ser classificadas como rápidas (tempo médio $<5$ dias); intermediárias (tempo médio $>5<10$ dias) e lentas (tempo médio $>10$ dias). Eremanthus 
incanus apresenta sementes intermediárias, enquanto E. elaeagnus e E. glomerulatus apresentam sementes lentas para germinação (sensu Ferreira et al. 2001).

A produção de aquênios em Eremanthus é alta, porém, muitos não se desenvolvem e parte deles é predada. Aproximadamente 70 e $56 \%$ dos aquênios de E. elaeagnus e E. glomerulatus, coletados em agosto e julho/2001, respectivamente (Tab. 1), eram aparentemente viáveis e, deste percentual, cerca de 91 e 73\% não possuíam embriões (Tab. 5). Maluf \& Wizentier (1998) também encontraram baixa percentagem de aquênios desenvolvidos $(35,4$ e 50,1\%) em relação ao número total de aquênios produzidos por capítulo, em diferentes populações de Eupatorium vauthierianum (Asteraceae).

As baixas percentagens de germinação das espécies investigadas, especialmente de E. elaeagnus e $E$. glomerulatus, refletiram os baixos percentuais de aquênios férteis produzidos. Muitas outras espécies de Asteraceae também apresentaram baixa germinabilidade (Cesarino \& Zaidan 1998; Chmielewski 1999; Falkner et al. 1997; Ferreira et al. 2001; Figueiredo 1975; Maluf 1993; Maluf \& Wizentier 1998; Ruggiero \& Zaidan 1997), sendo que alguns autores verificaram altas proporções de aquênios sem sementes (Clampitt 1987; Radford \& Cousens 2000; Sassaki et al. 1999; Walker \& Powell 1999), indicando que a baixa produção de aquênios férteis, resultando em baixa germinabilidade, ocorre com freqüência na família.

Byers (1995) investigou as causas da baixa produção de sementes em Eupatorium resinosum, uma Asteraceae auto-incompatível, e verificou que tanto a deposição de quantidade insuficiente de pólen como a incompatibilidade do pólen parecem contribuir com a baixa produção de sementes em pequenas populações desta espécie. Les et al. (1991) também verificaram a ocorrência de mecanismo de auto-incompatibilidade genética em uma população clonal de Aster furcatus (Asteraceae). Segundo Warburton et al. (2000), se existir pouca diversidade genética dentro de populações, e se o fluxo gênico entre populações não ocorrer, a existência de mecanismos de auto-incompatibilidade pode resultar em pouca ou nenhuma produção de frutos.

Espécies clonais freqüentemente produzem alta proporção de sementes inviáveis (Bell et al. 1993). Durante este estudo foi observado um indivíduo de $E$. elaeagnus, com dois ramos aéreos, $50 \mathrm{~cm}$ distantes um do outro e unidos por órgão subterrâneo horizontal, a cerca de $3 \mathrm{~cm}$ da superfície do solo. Foi observado também um indivíduo de E. erythopappus com cerca de 50 ramos aéreos unidos por órgão subterrâneo com características semelhantes ao observado para E. elaeagnus. Considerando a ocorrência de reprodução assexuada em espécies do gênero Eremanthus e que a auto-incompatibilidade é freqüente na família Asteraceae (Richards 1986), sugere-se que uma das possíveis causas da baixa produção de sementes viáveis nestas espécies pode ser o reflexo da baixa variabilidade genética e da presença de mecanismos de auto-incompatibilidade.

Os resultados obtidos mostram que a viabilidade das sementes variou não apenas entre diferentes populações (E. elaeagnus), como também entre períodos de produção em uma mesma população (E. elaeagnus e E. incanus). As variações temporais na viabilidade das sementes sugerem que outros fatores, como polinização insuficiente ou estresse ambiental, podem estar influenciando na reprodução sexuada destas espécies. Segundo Bell et al. (1993), fatores ambientais como limitação na disponibilidade de água durante o período de maturação das sementes podem reduzir a proporção de sementes viáveis produzidas.

Considerando-se a alta pressão antrópica à qual estão sujeitas E. elaeagnus, E. glomerulatus e E. incanus, a baixa produção de sementes férteis poderá ser um problema importante não só sob o ponto de vista ecológico, mas também por dificultar o cultivo destas espécies. Os resultados obtidos neste trabalho, bem como a realização de estudos que enfoquem outros aspectos da biologia reprodutiva e fatores ambientais que possivelmente estão interferindo na reprodução destas espécies, são indispensáveis para estabelecer estratégias para a conservação das mesmas.

\section{Agradecimentos}

As autoras agradecem ao Dr. José Pires de Lemos Filho, pelas sugestões apresentadas; ao Dr. Harold Robinson, pela identificação das espécies; ao Dr. Ivan Sampaio, pelo auxílio nas análises estatísticas; a Arabela V. Franco, pela revisão do Abstract.

\section{Referências bibliográficas}

Afolayan, A.J.; Meyer, J.J.M. \& Leeuwner, D.V. 1997. Germination in Helichrysum aureonitens (Asteraceae): effects of temperature, light, gibberellic acid, scarification and smoke extract. South African Journal of Botany 63(1): 22-24. 
Antunes, F.Z. 1986. Caracterização climática do estado de Minas Gerais. Informativo Agropecuário 12: 9-13.

Baskin, C.C. \& Baskin, J.M. 1988. Germination ecophysiology of herbaceous plant species in a temperate region. American Journal of Botany 75(2): 286-305.

Bell, D.T.; Plummer, J.A. \& Taylor, S.K. 1993. Seed germination ecology in southwestern western Australia. The Botanical Review 59: 24-73.

Bewley, J.D. \& Black, M. 1994. Seeds: Physiology of Development and Germination. New York, Plenum Press.

Bohlmann, F., Zdero, C., King, R.M. \& Robinson, H. 1980. Sesquiterpene lactones from Eremanthus species. Phytochemistry 19: 2663-2668.

Borghetti, F. \& Ferreira, A.G. 2004. Interpretação de resultados de germinação. In: A.G. Ferreira \& F. Borghetti (orgs.). Germinação: do básico ao aplicado. Porto Alegre, Artmed.

Bunker, K.V. 1994. Overcoming poor germination in Australian daisies (Asteraceae) by combinations of gibberellin, scarification, light and dark. Scientia Horticulturae 59: 243252.

Byers, D.L. 1995. Pollen quantity and quality as explanations for low seed set in small populations exemplified by Eupatorium (Asteraceae). American Journal of Botany 82(8): 10001006.

Cesarino, F. \& Zaidan, L.B.P. 1998. Vernonia cognata Less. (Asteraceae): armazenamento e viabilidade dos aquênios. Hoehnea 25(1): 59-70.

Chmielewski, J.G. 1999. Consequences of achene biomass, within-achene allocation patterns, and pappus on germination in ray and disc achenes of Aster umbellatus var. umbellatus (Asteraceae). Canadian Journal of Botany 77: 426-433.

Clampitt, C.A. 1987. Reproductive Biology of Aster curtus (Asteraceae), a pacific northwest endemic. American Journal of Botany 74: 941-946.

Corkidi, L.; Rincon, E. \& Vazquez-Yanes. 1991. Effects of light and temperature on germination of heteromorphic achenes of Bidens odorata (Asteraceae). Canadian Journal of Botany 69: 574-579.

Delouche, J.C.; Sill, T.W.; Raspet, M. \& Lienhard, M. 1962. The tetrazolium test for seed viability. Missouri Agriculture Experimental Station Technical Bulletin 51: 1-63.

Falkner, M.B.; Laven, R.D. \& Aplet, G.H. 1997. Experiments on germination and early growth of three rare and endemic species of Hawaiian Tetramolopium (Asteraceae). Biological Conservation 80: $39-47$.

Felippe, G. M. \& Silva, J. C. S. 1984. Estudos de germinação em espécies do cerrado. Revista Brasileira de Botânica 7(2): 157-163.

Fenner, M. 1983. Relationships between seed weight, ash content and seedling growth in twenty-four species of Compositae. New Phytologist 95: 697-706.

Ferreira, A.G.; Cassol, B.; Rosa, S.G.T.; Silveira, T.S.; Stival, A.L. \& Silva, A.A. 2001. Germinação de sementes de Asteraceae nativas no Rio Grande do Sul, Brasil. Acta Botanica Brasilica 15(2): 231-242.

Figueiredo, R.C.L. 1975. Notas preliminares sobre a germinação e ocorrência de derivados cumarínicos em aquênios de Eupatorium pauciflorum H.B.K. (Compositae). Hoehnea 5: 47-57.
Garcia, Q.S.; Sharif, R.R. 1995. Germinação e dormência em aquênios de Acanthospermum hispidum DC, uma espécie invasora. Revista Brasileira de Botânica 18(1): 17-25.

Gomes, V. \& Fernandes, G.W. 2002. Germinação de aquênios de Baccharis dracunculifolia D.C. (Asteraceae). Acta Botanica Brasilica 16(4): 421-427.

Grime, J.P.; Mason, G.; Curtis, A.V.; Rodman, J.; Band, S.R.; Mowforth, M.A.G.; Neal, A.M. \& Shaw, S. 1981. A comparative study of germination characteristics in a local flora. Journal of Ecology 69: 1017-1059.

Jusaitis, M.; Polomka, L. \& Sorensen, B. 2004. Habitat specificity, seed germination and experimental translocation of the endangered herb Brachycome muelleri (Asteraceae). Biological Conservation 106: 251-66.

Labouriau, L.G. 1983. A Germinação das Sementes. Washington D.C, Secretaria Geral da Organização dos Estados Americanos.

Ladeira, A.M.; Zaidan, L.B.P. \& Figueiredo-Ribeiro, R.C.L. 1987. Ageratum conyzoides L. (Compositae): Germinação, floração e ocorrência de derivados fenólicos em diferentes estádios de desenvolvimento. Hoehnea 14: 53-62.

Lemos Filho, J.P.; Guerra, S.T.M.; Lovato, M.B. \& Scotti, M.R.M.M.L. 1997. Germinação de sementes de Senna macranthera, Senna multijuga e Sthyphnodendron polyphyllum. Pesquisa Agropecuária Brasileira 32(4): 357-361.

Les, D.H.; Reinartz, J.A.; Esselman, E.J. 1991. Genetic consequences of rarity in Aster furcatus (Asteraceae), a threatened, self-incompatible plant. Evolution 45(7): 1641-1650.

Leishman, M.R.; Wright, A.T. \& Westoby, M. 2001. The evolutionary ecology of seed size. In: Fenner, M. Seeds: The Ecology of Regeneration in Plant Communities. Wallingford, UK, CAB International. $2^{\text {nd }} \mathrm{ed}$.

Macleish, N.F.F. 1987. Revision of Eremanthus (Compositae: Vernonieae). Annals of Missouri Botanical Garden 74: 265-290.

Maluf, A.M. 1993. Efeito da temperatura e tuz na germinação de sementes de duas populações de Vernonia polyanthes (Asteraceae). Hoehnea 20: 133-137.

Maluf, A.M. \& Wizentier, B. 1998. Aspectos fenológicos e germinação de sementes de quatro populações de Eupatorium vauthierianum DC. (Asteraceae). Revista Brasileira de Botânica 21(3): 247-251.

Mendonça, M.P. \& Lins, L.V. 2000. Lista Vermelha das Espécies Ameaçadas de Extinção da Flora de Minas Gerais. Belo Horizonte, Fundação Biodiversitas, Fundação Zoo-Botânica de Belo Horizonte.

Owens, D.W. \& Call, C.A. 1985. Germination characteristics of Helianthus maximilianai Schrad. and Simsia calva (Engelm. \& Gray) Gray. Journal of Range Management 38(4): 336-339.

Pons, T.L. 1992. Seed Response to Light. In: F. Fenner. Seeds: The Ecology of Regeneration in Plant Communities. Wallingford, UK, CAB International.

Picman, A.K. 1986. Biological activities of sesquiterpene lactones. Biochemical Systematics and Ecology 14(3): 255-281.

Radford, I.J. \& Cousens, R.D. 2000. Invasiveness and comparative life-history traits of exotic and indigenous Senecio species in Australia. Oecologia 125: 531-542. 
Randi, A.M. \& Felippe, G.M. 1981. Efeito de temperatura, luz e reguladores de crescimento na germinação de Stevia rebaudiana Bert. Ciência e Cultura 33(4): 404-411.

Richards, A.J. 1986. Plant Breeding Systems. London, UK, George Allen and Unwin.

Ruggiero, P.G.C. \& Zaidan, L.B.P. 1997. Estudos de desenvolvimento de Viguiera robusta Gardn., uma Asteraceae do cerrado. Revista Brasileira de Botânica 20(1): 1-9.

Sampaio, I.B.M. 2002. Estatística Aplicada à Experimentação Animal. Belo Horizonte, FEPMVZ Ed. Escola de Veterinária, Universidade Federal de Minas Gerais.

Sassaki, R.M.; Rondon, J.N.; Zaidan, L.B.P. \& Felippe, G.M. 1999. Germination of seeds from herbaceous plants artificially stored in cerrado soil. Revista Brasileira de Biologia 59(2): 271-279.

Schütz, W. \& Milberg, P. 1997. Seed germination in Launaea arborescens: a continuously flowering semi-desert shrub. Journal of Arid Environments 36: 113-122.
Tamashino, J.Y. \& Leitão-Filho, H.F. 1978. Observações sobre o ciclo de vida de Bidens pilosa L. (Compositae Heliantheae). Hoehnea 7: 27-40.

Thompson, P.A. 1974. Effects of fluctuating temperatures on germination. Journal of Experimental Botany 25(84): 164-175

Thompson, K. \& Grime, J.P. 1983. A comparative study of germination responses to diurnally-fluctuating temperatures. Journal of Applied Ecology 20: 141-156.

Vázquez-Yanes, C. \& Orozco-Segovia, A. 1996. Physiological ecology of seed dormancy and longevity. In: S.S. Mulkey; R.L. Chazdon \& A.P. Smith. Tropical Forest Plant Ecophysiology. New York, Chapman \& Hall.

Walker, L.R. \& Powell, E.A. 1999. Regeneration of the Mauna Kea silversword Argyroxiphium sandwicense (Asteraceae) in Hawaii. Biological Conservation 89: 61-70.

Warburton, C.L.; James, E.A.; Fripp, Y.J.; Trueman, S.J. \& Wallace, H.M. 2000. Clonality and sexual reproductive failure in remnant populations of Santalum lanceolatum (Santalaceae). Biological Conservation 96: 45-54. 